\title{
Fração prevenível na associação entre a atividade física e doença cardiovascular: uma revisão narrativa
}

\author{
Preventable fraction in the association between physical activity and cardiovascular \\ disease: a narrative review
}
Fracción prevenible en la asociación entre la actividad física y la enfermedad cardiovascular: una revisión narrativa

\begin{abstract}
Vitoria Vilas Boas da Silva Bomfim ${ }^{1 *}$, Emilie dos Santos Aleluia ${ }^{1}$, Heloisa Ferreira de Jesus ${ }^{1}$, Amanda Larissa de Souza Matos de Almeida', Gislana de Souza Costa', Adriele da Silva Brito', Tainara Regina Castro Silva ${ }^{1}$, Leidiane Araújo Oliveira', Hytirsia Santos Almeida', Naiara Caroline da Silva Barreto².
\end{abstract}

\section{RESUMO}

Objetivo: Revisar na literatura o papel da atividade física na prevenção de eventos cardiovasculares. Revisão Bibliográfica: Os fatores modificáveis ou controláveis são aqueles que podem ser revertidos a partir de uma mudança nos hábitos de vida do paciente. São eles: tabagismo, hipertensão arterial, diabetes mellitus, dislipidemia, obesidade e sedentarismo. O tabagismo é fator de risco que pode ser totalmente eliminado, apesar de ser difícil. Estudos mostram que a redução do nível pressórico traz benefícios diretos na redução da morbimortalidade por DCV. Evidências mostram que o risco de DCV está aumentando em pacientes com DM, sendo que a DM 2 apresenta um risco duas vezes maior para homens e quatro vezes maior para mulheres. A prática regular de exercícios físicos está diretamente relacionada com a prevenção e o controle das doenças cardiovasculares (DCV), uma vez que ela exerce influência nos fatores de risco modificáveis para cada tipo de artigo usar o subtítulo pertinente. Considerações Finais: $O$ estudo demonstrou então que pacientes mais ativos têm um risco de DCV reduzido em aproximadamente $30 \%$ a $40 \%$, quando comparado com aqueles menos ativos, evidenciando uma relação inversa entre atividade física e risco de DCV.

Palavras-chave: Doenças cardiovasculares, Exercício físico, Prevenção de doenças.

\begin{abstract}
Objective: To review in the literature the role of physical activity in the prevention of cardiovascular events. Bibliographic Review: The modifiable or controllable factors are those that can be reversed from a change in the patient's lifestyle. They are: smoking, high blood pressure, diabetes mellitus, dyslipidemia, obesity and physical inactivity. Smoking is a risk factor that can be completely eliminated, although it is difficult. Studies show that the reduction in blood pressure level has direct benefits in reducing morbidity and mortality due to CVD. Evidence shows that the risk of CVD is increasing in patients with DM, with DM 2 presenting a twice as high risk for men and four times as high for women. Regular physical exercise is directly related to the prevention and control of cardiovascular diseases (CVD), since it influences the modifiable risk factors for each type of article using the relevant subtitle. Final Considerations: The study then showed that more active patients have a reduced risk of CVD by approximately $30 \%$ to $40 \%$, when compared to those less active, showing an inverse relationship between physical activity and CVD risk.
\end{abstract}

Keywords: Cardiovascular disease, Exercise, Disease prevention.

${ }^{1}$ Centro Universitário Jorge Amado (UNIJORGE), Salvador - BA. *E-mail: vitoriavilasboas31@gmail.com

2 Universidade Salvador (UNIFACS), Salvador - BA. 


\section{RESUMEN}

Objetivo: Revisar en la literatura el papel de la actividad física en la prevención de eventos cardiovasculares. Revisión Bibliográfica: Los factores modificables o controlables son aquellos que pueden revertirse a partir de un cambio en el estilo de vida del paciente. Ellos son: tabaquismo, hipertensión arterial, diabetes mellitus, dislipidemia, obesidad e inactividad física. Fumar es un factor de riesgo que puede eliminarse por completo, aunque es difícil. Los estudios muestran que la reducción de la presión arterial tiene beneficios directos en la reducción de la morbilidad y mortalidad por ECV. La evidencia muestra que el riesgo de ECV está aumentando en pacientes con DM, con DM 2 presentando un riesgo dos veces mayor para los hombres y cuatro veces más alto para las mujeres. El ejercicio físico regular está directamente relacionado con la prevención y el control de las enfermedades cardiovasculares (ECV), ya que influye en los factores de riesgo modificables para cada tipo de artículo utilizando el subtítulo correspondiente. Consideraciones Finales: El estudio mostró que los pacientes más activos tienen un riesgo reducido de ECV en aproximadamente un $30 \%$ a un $40 \%$, en comparación con los menos activos, mostrando una relación inversa entre la actividad física y el riesgo de ECV.

Palabras clave: Enfermedades Cardiovasculares, Ejercicio físico, Prevención de enfermedades.

\section{INTRODUÇÃO}

As doenças cardiovasculares (DCV) são um grupo de doenças do coração e dos vasos sanguíneos no qual ocorre uma falha na sua função normal em um dos seus mecanismos, seja por falência na bomba, no fluxo regurgitante e colateral, distúrbio na condução cardíaca, ruptura do coração ou vaso sanguíneo, ou por bloqueio que impede que o sangue flua no corpo, geralmente ocasionado por acúmulo de gordura nas paredes internas dos vasos sanguíneos que irrigam o coração (OPAS, 2017).

Vários são os fatores de risco associados aumento de doenças cardiovasculares, podendo ser de causas modificáveis e não modificáveis. Os principais fatores de risco modificáveis são hipertensão, obesidade, sedentarismo, tabagismo, etilismo, dislipidemias e altos níveis de glicose sanguínea (SCHMIDT ML, et al., 2011; PELLANDA LC, 2011).

As doenças cardiovasculares (DCV) são a maior causa de morbimortalidade no Brasil e no mundo, sendo responsáveis por aproximadamente $20 \%$ dos óbitos em pessoas com mais de 30 anos (MANSUR AP, FAVARATO D, 2011; BAENA CP, 2013; ISHITANI LH, et al., 2016). Apesar de a DCV ser a principal causa de morte em ambos os países desenvolvidos e subdesenvolvidos, a maior parte dos acometidos vem dos países subdesenvolvidos (BAENA CP, 2013). Grande parte desse impacto, advém da mortalidade precoce da população e dos gastos econômicos no tratamento dos portadores de DCV, o que vem a se tornar um grande impasse para o desenvolvimento socioeconômico desses países (BAENA CP, 2013; GOLDMAN L, et al., 2017).

As duas principais doenças cardiovasculares são a doença isquêmica do coração (DIC) e as doenças cerebrovasculares, isto porque elas são responsáveis, respectivamente por 95.449 e 97.860 mortes no Brasil, já as doenças cardiovasculares ateroscleróticas vêm em terceiro lugar com 193.309 mortes, segundo dados do Ministério da Saúde (ISHITANI LH, et al., 2016; MANSUR AP e FAVARATO D, 2011).

As DCVs apresentaram uma grande elevação da mortalidade no Brasil entre as décadas de 30 e 80 , período que com o incremento do processo de industrialização do país, tendo então uma queda após a década de 80 (BAENA CP, 2013; ISHITANI LH, et al., 2016; MANSUR AP e FAVARATO D, 2011; GOLDMANL, et al., 2017). Atualmente o risco de morte por DCV apresenta uma tendência decrescente, como foi demonstrado no estudo de Mansur e Favarato (BARRAG FH e CUEVAS RT, 2016).

Neste estudo os autores fizeram uma atualização das taxas de mortalidade por DCV no Brasil de 1980 a 2012, constatando que nesse período houve uma redução de morte por doença isquêmica do coração (DIC) e doenças cerebrovasculares (DCbV) - os dois principais tipos de doenças cardiovasculares - sendo essa queda maior na região sudeste do país e para as DCbVs. Constatou-se ainda que os níveis de mortalidade por DIC e DCbV no Brasil "estão entre os mais altos do mundo" e assemelham-se ao de países como a China e os do Leste Europeu (MANSUR AP e FAVARATO D, 2011). 
Apesar da tendência a diminuir, o risco de morte por DCV ainda apresenta-se bastante elevado no Brasil, o que se pode atribuir a uma grande prevalência dessas morbidades (BAENA CP, 2013; ISHITANI LH, et al., 2016; MANSUR AP e FAVARATO D, 2011; GOLDMAN L, et al., 2017). Essa grande prevalência é também observada em países em desenvolvimento e pode estar tanto associada a um mau controle dos fatores de risco e consequente falha na prevenção, assim como se associa ao nível socioeconômico do indivíduo (ISHITANI LH, et al., 2016; MANSUR AP e FAVARATO D, 2011). Lenice Harumi Ishitani em seus estudos constatou que os níveis socioeconômicos estão inversamente relacionados com a mortalidade por DCV no Brasil (RIQUE ABR, et al., 2010).

Isto é, quanto menores a escolaridade, a renda e o nível de pobreza de uma determinada população, maior era a taxa de mortalidade por DCV. Além disso, trouxeram também que o risco de morte por doença cardiovascular também está associado com o nível de escolaridade e as condições de moradia, sendo essa relação também inversamente proporcional. Essas relações podem ser explicadas pelo baixo nível de informação sobre prevenção e controle de fatores de risco das DCVs obtido por essa população de menor nível socioeconômico. Dessa forma, eles ficam mais vulneráveis à ocorrência dessas doenças (ISHITANI LH, et al., 2016).

Já em relação ao mundo, têm-se evidências de que em países desenvolvidos foi encontrada a mesma relação entre níveis socioeconômicos e DCV vista no Brasil (ISHITANI LH, et al., 2016; MANSUR AP, FAVARATO D, 2011). Alguns estudos ecológicos trazem que nos Estados Unidos da América (EUA), Austrália e em municípios como Rio de Janeiro e São Paulo, o maior nível socioeconômico está associado com uma menor mortalidade por DCV (ISHITANI LH, et al., .2016).

Esse fato pode estar vinculado ao fato de que indivíduos com maior renda têm um melhor acesso a bens e serviços. Além disso, pacientes com maior poder aquisitivo, em geral, têm maior escolaridade e maior acesso à informação, podendo então prevenir as DCV através de medidas de modificação dos fatores de riscos associados à doença (ISHITANI LH, et al., 2016; MANSUR AP e FAVARATO D, 2011).

Frente ao exposto, o estudo revisa na literatura o papel da atividade física na prevenção de eventos cardiovasculares.

\section{REVISÃO BIBLIOGRÁFICA}

\section{Fatores de risco para doenças cardiovasculares}

Os fatores de risco para as doenças cardiovasculares podem ser classificados entre modificáveis e não modificáveis. Os não modificáveis são aqueles que são essencialmente imutáveis como: idade, sexo, história familiar, predisposição genética. Em contrapartida, os fatores modificáveis ou controláveis são aqueles que podem ser revertidos a partir de uma mudança nos hábitos de vida do paciente. São eles: tabagismo, hipertensão arterial, diabetes mellitus, dislipidemia, obesidade e sedentarismo. O conhecimento a fundo desses fatores é essencial para a realização de práticas preventivas evitando o desenvolvimento das doenças cardiovasculares (GOLDMAN L, et al., 2017; ANDREA M, et al., 2010; SHIROMA EJ e LEE L, 2010; SIMON HB, 2015).

\section{Tabagismo}

Assim como a dislipidemia e a hipertensão, o tabagismo é considerado um dos três principais fatores de risco para DCV. O tabagismo é considerado uma doença neuro comportamental devido à dependência a nicotina e está relacionado a grande parte das mortes por doenças não transmissíveis, o ato de fumar aumenta os níveis de triglicerídeos e lipoproteínas de baixa densidade (LDL) no organismo que podem levar a resistência à insulina e infecções crônicas, provoca disfunções vasculares, arteriosclerose, trombose e aumento do estresse oxidativo, fatores esses que predispõem ao desenvolvimento de DCV (RIQUE ABR, et al., 2010; GOLDMAN L, et al., 2017).

O indivíduo que é fumante passivo tem uma maior probabilidade de ir a óbito, uma vez que é a $3^{\circ}$ causa de morte evitável no mundo segundo o Instituto nacional de câncer. A exposição dessas pessoas a substâncias tóxicas da fumaça pode levar ao desenvolvimento de inúmeras doenças, sendo algumas delas cardiovasculares, estudos mostram que o hábito de fumar em jovens pode levar aumentar a incidência de aterosclerose outro fator de risco para o desenvolvimento da DCV (SILVA LCC, et al., 2016). 
O tabagismo é fator de risco que pode ser eliminado, apesar de ser difícil. A eliminação do hábito de fumar por, pelo menos, dois anos pelo paciente reduz a incidência de doença arterial coronariana (DAC) para níveis iguais ao de pacientes não fumantes. Por isso destaca-se a importância de se parar de fumar já que as diversas substâncias tóxicas no organismo podem levar a doenças crônicas e fatais (GOLDMAN L, et al., 2017).

\section{Hipertensão arterial}

A hipertensão arterial é uma das patologias de maior prevalência na população adulta e principalmente nos idosos. A hipertensão arterial é definida como uma elevação da pressão sistólica acima de $140 \mathrm{mmHg} e$ da diastólica acima de $90 \mathrm{mmHg}$. Está associada ao aumento na morbidade-mortalidade por todas as causas cardiovasculares e os programas de exercício estão associados à prevenção do desenvolvimento de hipertensão e também à restrição da pressão arterial (PA), tanto em normotensos quanto em hipertensos. Estudos mostram que a redução do nível pressórico traz benefícios diretos na redução da morbimortalidade por DCV (RIQUE ABR, et al., 2010).

Dentre as DCVs, a Hipertensão Arterial Sistêmica (HAS) é um fator de risco de grande importância para complicações cardíacas e cerebrovasculares, tida como um problema de saúde pública em domínio mundial. Em 2000, a predominância da HAS na população mundial era de $25 \%$ e a suposição para o ano de 2025 é de $29 \%$. Estudos realizados no Brasil mostra que a prevalência da hipertensão variou entre 22,3 e 43,9\%, com média de 32,5\% (RIQUE ABR, et al., 2010).

Quase todas as nações, a prevenção e o controle da HAS trazem importantes encadeamento, e a utilização de novas estratégias e abordagens que especifiquem com mais clareza os indivíduos em situação de risco, ofertar benefícios tanto para o indivíduo com hipertensão como para a sociedade (RADOVANOVIC CAT, et al., 2014).

No entanto, por ser uma doença crônica, o monitoramento da HAS requer acompanhamento e tratamento por toda a vida. Para alcançar o controle preciso da pressão arterial dos pacientes hipertensos se faz necessário o acompanhamento com um médico e tratamento farmacológico (GOLDMAN L, et al., 2017; RIQUE ABR, et al., 2010).

\section{Diabetes mellitus}

É definida como uma desordem metabólica, sendo classificado em Diabetes mellitus tipo 1 e 2 (DM 1 e 2). Evidências mostram que o risco de DCV está aumentando em pacientes com diabetes mellitus, sendo que a diabetes mellitus 2 apresenta um risco duas vezes maior para homens e quatro vezes maior para mulheres (GOLDMAN L, et al., 2017).

Pacientes com essa morbidade geralmente apresenta outra comorbidades como: hipertensão arterial, altos níveis de triglicérides e menores níveis de HDL (GOLDMAN L, et al., 2017; RIQUEABR, et al., 2010). Esses pacientes são classificados como portadores de síndrome metabólica, e o controle medicamentoso ou através da mudança de hábitos de vida dessas comorbidades é essencial para reverter o diabetes e, principalmente, o risco para DCV (GOLDMAN L, et al., 2017; SHIROMA EJ e LEE I, 2010).

Com o envelhecimento, o aumento da urbanização, o sedentarismo e a alimentação indevida, o Diabetes Mellitus se tornou uma epidemia mundial. Seus sintomas mais clássicos são os 4 P's sendo eles Poliúria, polidpisia, polifalgia, e perda de peso além de fraqueza, letargia, prurido cutâneo e vulvar. Seu diagnóstico se faz através de exame laboratorial, teste oral de tolerância a glicose e glicemia casual. Seu tratamento se dá através de mudanças no estilo de vida e farmacoterapia (BRASIL, 2013).

A prática de atividade física é indicada para os pacientes diagnosticados com o Diabetes Mellitus independente do seu tipo, pois ajuda na perda de peso diminuindo o risco de doenças cardiovasculares melhorando a sua qualidade de vida. Essa atividade física deve ser iniciada aos poucos com pequenas caminhadas e aumentando seu tempo aos poucos. Usando sapatos confortáveis e está alerta a hipoglicemia, levando consigo um alimento rico em carboidratos além de ingerir bastante líquido (BRASIL, 2013). 


\section{Dislipidemia}

É caracterizada pelo aumento dos níveis séricos do colesterol total (CT), Lipoproteína de Baixa Densidade (LDL colesterol) e triglicérides e queda dos níveis de Lipoproteína de Alta Densidade (HDL colesterol) (GOLDMAN L, et al., 2017). O controle dos níveis desses fatores a níveis ótimos está relacionado à eliminação do fator de risco para o desenvolvimento de doenças cardiovasculares (GOLDMAN L, et al., 2017; ANDREA M, et al., 2010; SHIROMA EJ e LEE I, 2010; SIMON HB, 2015).

Dentre as dislipidemias, a classificação fenotípica ou bioquímica considera os valores de Colesterol total, LDL- C, triglicérides e HDL- C que compreende quatro tipos bem definidos: hipercolesterolemia isolada: elevação isolada do LDL- C; hipertrigliceridemia isolada: elevação isolada dos triglicérides; hiperlipidemia mista: aumento de LDL-C e triglicérides; HDL-C baixo: redução do HDL-c isolada ou em associação ao aumento de LDL-C ou de TG (XAVIER HT, et al., 2013).

A hipercolesterolemia sendo em si assintomática, o aumento do colesterol mesmo sendo a longo prazo pode causar aterosclerose. No decorrer de um período, os níveis altos de colesterol colaboram para a formação de placas ateromatosas nas artérias. Isso pode provocar estenose progressiva, podendo causar diminuição ou bloqueio total das artérias vinculadas. As placas menores podem também ocasionar a produção de um coágulo que impede a passagem do sangue. Uma interrupção súbita de uma artéria coronária pode causar um ataque cardíaco ou enfarte do miocárdio. Uma obstrução de uma artéria que irriga o cérebro possivelmente pode ocasionar um acidente vascular cerebral (FINN AV, et al., 2010).

Se a evolução desse estreitamento ou bloqueio for progressivo, a distribuição de sangue aos tecidos e órgãos diminuirá pouco a pouco até provocar a insuficiência desse órgão. Portanto, esta limitação de fornecimento de sangue (isquemia) pode mostrar-se por meio de sintomas através específicos (FINN AV, et al., 2010).

A hipertrigliceridemia (HTG) procede do aumento das lipoproteínas responsáveis pela condução de triglicérides (TG). É constantemente secundária ao aumento da ingestão de álcool, obesidade, diabetes, como um efeito adverso de medicamento. O aumento acelerado dos níveis de TG pode ocasionar DCV (FRANCISCO AR, et al., 2016).

As hiperlipidemias são modificações metabólicas que acontecem quando níveis de lípidos presentes na corrente sanguínea estão altos (BEVILAQUA MR, 2007). Assim então, podendo ocasionar DCV. A redução dos níveis de lipoproteínas de alta densidade (HDL), e a elevação dos triglicerídeos impacta no aumento da pressão arterial, tornando-se importante fator de risco para DCV (ROCHA FL, et al., 2013).

Sabe-se que as LDL-c são as maiores carreadoras de colesterol para as células, enquanto as HDL-c, participam do transporte reverso do colesterol, sendo este papel de extrema importância, pois possui função anti-aterogênicas. As dislipidemias na infância e na adolescência adquiriram maior importância entre as patologias mais estudadas neste século pelas repercussões que trazem na vida adulta (FAGHERAZZI S, et al., 2008).

\section{Obesidade}

A obesidade está associada com o acúmulo excessivo de gordura corporal e pode ser calculada a partir de medidas antropométricos de índice de massa corpórea (IMC), a circunferência da cintura (CC) é utilizada para avaliar o risco de complicações metabólicas associadas à obesidade (GOLDMAN L, et al., 2017; ANDREA M, et al., 2010; SIMON HB, 2015).

O primeiro está relacionado com a distribuição da gordura corporal e indica um maior risco de desenvolvimento de doenças crônicas. Já a CC está relacionada com a quantidade de tecido adiposo visceral, estando mais fortemente relacionada com um maior risco de desenvolver DCV (SHIROMA EJ, LEE I, 2010).

O excesso de peso ainda predispõe o indivíduo a outras comorbidades como hipertensão arterial, resistência à insulina, diabetes mellitus 2, hipertrigliceridemia, entre outros. Sendo assim, um controle rigoroso da quantidade de gordura corporal é essencial para a eliminação do risco de desenvolver DCV (GOLDMAN L, et al., 2017; ANDREA M, et al., 2010; SHIROMA EJ, LEE I, 2010; SIMON HB, 2015). 
O balanço energético é a igualdade alcançado com base no total de calorias ingeridas, e o total de calorias gasta pelo corpo na realização de suas atividades físicas. O gasto calórico, a outra parte da equação de balanço energético, é resultado de :taxa metabólica basal (TMB), que é a quantidade de calorias que o corpo necessita para manter-se nutridos durante o decorrer das atividades diárias; ação específica dos alimentos, que correspondente aos gastos calóricos necessário para a digestão, absorção; e atividade física movimento corporal produzido pela musculatura esquelética que resulte em gasto calóricos (GOLDMAN L, et al., 2017; RIQUE ABR, et al., 2010; SHIROMA EJ, LEE I, 2010).

A diminuição da produção de atividade física e sua relação com a elevação na prevalência da obesidade refere-se às alterações na distribuição dos trabalhos por setores e nos processos de redução do esforço físico no trabalho ocupacional (MENDONÇA CP e ANJOS LAD, 2004).

\section{Sedentarismo}

A inatividade física é atualmente considerada uma pandemia, uma vez que um terço da população mundial não cumpre as recomendações mínimas de atividade física. $O$ ato de praticar atividade físicas está relacionado como um fator de proteção contra doenças cardiovasculares, pois está diretamente relacionada com alterações em fatores de risco já supracitados como obesidade, dislipidemia, hipertensão e diabetes mellitus (GOLDMAN L, et al., 2017; RIQUE ABR, et al., 2010; ANDREA M, et al., 2010; SHIROMA EJ e LEE I, 2010).

A revolução industrial trouxe uma nova dinâmica social, principalmente no que tange ao significativo aumento da carga horária de trabalho, o que ocasionou na alteração da rotina diária das pessoas, que sem muito tempo disponível procuraram cada vez mais fazer refeições rápidas através de fast foods, contribuindo para elevação dos índices dos problemas de saúde causados pelo excesso de açúcar, sal e gorduras aliados ao sedentarismo (FREITAS MM, et al., 2018).

Está má alimentação, pobre em nutrientes e vitaminas associada à inatividade física, observando que uma relevante parte da população não dispõe de tempo suficiente para realizar algum tipo de atividade física, tem trazido consequências para o corpo, que tem sido acometido de distúrbios metabólicos, cardiovasculares, dentre outros. O quantitativo de calorias ingeridas através dos alimentos hipercalóricos que a indústria alimentícia nos seduz a consumir é superior a energia gasta durante o dia, para realizarmos nossas atividades (FREITAS MM, et al., 2018).

\section{Exercício físico e doenças cardiovasculares}

A prática regular de exercícios físicos está diretamente relacionada com a prevenção e o controle das doenças cardiovasculares (DCV), uma vez que ela exerce influência nos fatores de risco modificáveis (GOLDMAN L, et al., 2017; ANDREA M, et al., 2010). Além disso, uma rotina de atividade física traz mudanças fisiológicas advindas do envelhecimento e promovem uma maior longevidade ao indivíduo (SIMON HB, 2015).

No âmbito da modificação dos fatores de risco para DCV, a literatura traz que praticar exercícios físicos aeróbios ou de resistência e intensidades leves a moderadas promove perda de peso, melhora no perfil lipídico, redução dos níveis sanguíneos de glicose, melhora da resistência à insulina e dos níveis pressóricos (GOLDMAN L, et al., 2017; ANDREAM, et al., 2010). Mostrando a importância da atividade física na prevenção para doenças cardiovasculares ao comprovar a modificação dos fatores de risco para DCV a partir da prática de atividade física.

Além disso, a American Heart Association, reconheceu a inatividade física como um importante fator de risco para DCV, conferindo um aumento da importância da prática de exercícios para prevenir DCV, uma vez que ele passou a ser um fator de risco propriamente dito, quanto antes sua importância era apenas relacionada à modificação de fatores de risco (SIMON HB, 2015).

Tendo em vista, é importante então se determinar qual a intensidade, o tipo e a duração da atividade física promovem benefícios à saúde. O American College of Sports Medicine (ACMS) traz em suas recomendações que é necessário um mínimo de 150-300 minutos, ou um gasto maior que 2000 kcal por semana, de prática de exercícios semanais de intensidade moderada para que se tenham benefícios relacionados a prevenção de DCV (RIQUE ABR, et al., 2010). 
Já o Physical Activity Guidelines for Americans recomenda atividades aeróbicas de moderada intensidade com tempo maior a 150 minutos semanais e atividades aeróbicas vigorosas de tempo maior que 75 minutos semanais, ou uma combinação equivalente a essa de exercícios aeróbicos moderados e intensos (OMURA J, et al., 2013).

Paffenbarger em um de seus estudos, realizado com alunos de Harvard, trouxe que o risco de infarto agudo do miocárdio reduz progressivamente quando o gasto energético com exercícios físicos era de 2000$3000 \mathrm{kcal}$ (quilocalorias) por semana (OMURA J, et al., 2013).

Além dele Morris et al trouxe em seus estudos com motoristas de ônibus em 1953 a prática de atividade física está relacionada com um fator de proteção para o desenvolvimento de doença arterial coronariana (DAC) (SIMON HB, 2015).

Nesse estudo o autor observou que motoristas de ônibus de Londres que passavam suas horas de trabalho andando pelos corredores dos ônibus e subindo e descendo as escadas dos ônibus com dois andares tinham um risco de desenvolver DAC menor do que aqueles que ficavam o tempo inteiro sentados (SIMON HB, 2015; OMURA J, et al., 2013).

Um outro estudo que acompanhou 55.137 adultos americanos durante quinze anos constatou que aqueles indivíduos que têm costume de praticar corridas têm de 30 a $45 \%$ menos risco de desenvolver ou morrer por DCV, além de terem 3 anos a mais de expectativa de vida, quando comparados com aqueles que não praticam corridas (OMURA J, et al., 2013). Todos esses estudos trazem evidências que explicam as recomendações feitas pela ACMS e pelo Physical Activity Guidelines for Americans.

Shiroma e Lee fizeram uma revisão que avaliou todos os estudos incluídos no Physical Activity Guidelines for Americans, além de outros estudos mais recentes. Os autores observaram então a associação entre atividade física e DCV, a segunda procurava avaliar a existência de uma relação entre dose-resposta, a terceira questão buscava avaliar se a atividade física pode melhorar o alto risco de DCV em indivíduos obesos (SIMON HB, 2015).

\section{CONSIDERAÇÕES FINAIS}

O estudo demonstrou que pacientes mais ativos têm um risco de DCV reduzido em aproximadamente $30 \%$ a 40\% quando comparado com aqueles menos ativos, evidenciando uma relação inversa entre atividade física e risco de DCV, a comparação segue também entre homens e mulheres no qual as mulheres foram um pouco mais pronunciadas, em relação aos jovens e idosos e com diferentes raças não houve alteração significativa.

\section{REFERÊNCIAS}

1. ANDREA M, et al. Identificação de Risco Cardiovascular em Pacientes Atendidos em Ambulatório de Nutrição. Rev Bras Cardiol, 2010; 23(2): 116-123.

2. BAENA CP. Doença Cardiovascular: Tendência de Mortalidade no Brasil e Prevenção Global. (Doutorado). Pontifícia Universidade Católica do Paraná, Curitiba, 2013; 102.

3. BARRAG FH, CUEVAS RT. Participantes de un programa masivo, 2016; 16(28): 949-962.

4. BEVILACQUA MR, et al. Hiperlipidemias e fatores dietéticos: estudo transversal entre nipo-brasileiros.Arq Bras Endocrinol Metab, 2007; 51(4): 547-558.

5. BRASIL. Ministério da saúde. Secretaria de Atenção à saúde. Departamento de Atenção Básica. Diabetes Mellitus. Caderno de Atenção Básica. Brasília DF. 2013

6. FAGHERAZZI S, et al. Impacto do exercício físico isolado e combinado com dieta sobre os níveis séricos de HDL, LDL, colesterol total e triglicerídeos.Rev. bras. med. esporte, 2008; 14(4): 381-386.

7. FINN AV, et al. Concept of vulnerable/unstable plaque. Arterioscler. Thromb. Vasc. Biol., 2010; 30(7): $1282-92$.

8. FRANCISCO AR, et al . Hipertrigliceridemia: há um papel para a aferese profilática? Relato de um caso. J. Bras. Nefrol., 2016; 38(3): 366-369.

9. FREITAS MM, et al. Prevalência e fatores associados à inatividade físicano tempo livre em adultos na cidade de Lauro de Fraitas- Bahia-Brasil. Revista Brasileira de Ciências da Saúde, 2018; 22(1): 27-36.

10. GOLDMAN L, et al. Tratado de medicina interna. 25ed. España: Elsevier; 2017; 762-774.

11. ISHITANI LH, et al. Desigualdade social e mortalidade precoce por doenças cardiovasculares no Brasil. Rev. Saúde Pública, 2006; 40(4): 684-691.

12. MANSUR AP, FAVARATO D. Mortalidade por doenças cardiovasculares no Brasil e na região Metropolitana de São Paulo: atualização 2011. Arq Bras Cardiol. 2012; 99(2): 755-61. 
13. MENDONCA CP, ANJOS LAD. Aspectos das práticas alimentares e da atividade física como determinantes do crescimento do sobrepeso/obesidade no Brasil. Cad. Saúde Pública, 2004; 20(3): 698-709.

14. OMURA J, et al. Adults eligible for Cardiovascular Disease Prevention Counseling and Participation in Aerobic physical Activity — United States. Morbidity and Mortality Weekly Report, 2013; 64(37):1047 1051.

15. OPAS - Organização Pan-Americana de Saúde. Doenças cardiovasculares. Brasil; 2017.

16. PELLANDA LC. Determinantes precoces das doenças cardiovasculares no curso da vida: uma mudança de paradigma para a prevenção. Rev Assoc Med Bras. 2011; 57(6): 6089.

17. RADOVANOVIC CAT, et al .Hipertensão arterial e outros fatores de risco associados às doenças cardiovasculares em adultos. Rev. Latino-Am. Enfermagem, 2014; 22(4): 547-553.

18. RIQUE ABR, et al. Nutrição e exercício na prevenção e controle das doenças cardiovasculares.Rev Bras Med esporte, 2010; 8(6): 244-254.

19. ROCHA FL, et al. Correlação entre indicadores de obesidade abdominal e lipídeos séricos em idosos.Rev. Assoc. Med. Bras., 2013; 59(1): 48-55.

20. SCHMIDT MI, et al. Chronic non-communicable diseases. Lancet. 2011; 377(9781): 194961.

21. SHIROMA EJ, LEE I. Exercice in cardiovascular disease physical activity and cardiovascular health lessons learned from epidemiological studies across age, gender, and race/ethnicity. Circulation, 2010; 122(7): 743-752.

22. SILVA LCC, et al . Controle do tabagismo: desafios e conquistas. J. bras. pneumol., 2016; 42(4): $290-298$.

23. SIMON HB. Exercise and health: Dose and response, considering both ends of the curve. The American Journal of Medicine, 2015; 128(11): 1171-1177.

24. XAVIER HT, et al. V Diretriz brasileira de dislipidemias e prevenção da aterosclerose.Arquivos brasileiros de cardiologia, 2013; 101(4): 1-20. 